Naho Endo-Kawamura, Mana Obata-Yasuoka*, Hiroya Yagi, Rena Ohara, Yuko Nagai, Miyuki Mayumi, Kanako Abe and Hiromi Hamada

\title{
Higher D-dimer level in the early third trimester predicts the occurrence of postpartum hemorrhage
}

DOI 10.1515/jpm-2015-0287

Received August 14, 2015. Accepted November 23, 2015.

\section{Abstract}

Aims: This study aimed to determine effective predictive factors for primary postpartum hemorrhage (PPH) among clinical blood parameters associated with coagulation and fibrinolysis and demographic characteristics.

Methods: We retrospectively studied 1032 women who underwent determinations of clinical blood parameters at gestational week (GW) 29-32 and GW 35-37 and gave birth to singleton infants at our hospital between January 2011 and December 2013. PPH was defined as estimated blood loss $\geq 700 \mathrm{~mL}$. Multivariate logistic regression analyses were used to determine independent risk factors and odds ratios (OR) for $\mathrm{PPH}$.

Results: PPH occurred in 104 of 1032 women (10\%). Three blood variables, fibrinogen level <4.0 g/L (OR [95\% CI], 1.96 [1.18-3.27]), antithrombin activity $<85 \%$ of normal activity level (1.84 [1.05-3.21]), and D-dimer level $>2.7 \mu \mathrm{g} / \mathrm{mL}$ (2.03 [1.29-3.19]) at GW 35-37, and three demographic characteristics, maternal age $\geq 35$ years (1.75 [1.15-2.68]), BMI $>28.2 \mathrm{~kg} / \mathrm{m}^{2}$ on admission for childbirth (1.95 [1.203.16]), and previous cesarean delivery (2.77 [1.31-5.83]), were identified as independent risk factors for $\mathrm{PPH}$.

Conclusion: Among blood parameters, higher D-dimer levels and lower levels of antithrombin activity and fibrinogen in late gestation were independent risk factors for PPH.

Keywords: Antithrombin; d-dimer; fibrinogen; postpartum hemorrhage.

*Corresponding author: Mana Obata-Yasuoka, MD, PhD, Faculty of Medicine, Department of Obstetrics and Gynecology, University of Tsukuba, 1-1-1 Tennodai, Tsukuba, Ibaraki 305-8575, Japan, Tel.: +81-29-853-3210, Fax: +81-29-853-3072,

E-mail: manobata@md.tsukuba.ac.jp

Naho Endo-Kawamura, Hiroya Yagi, Rena Ohara, Yuko Nagai, Miyuki Mayumi, Kanako Abe and Hiromi Hamada: Faculty of Medicine, Department of Obstetrics and Gynecology, University of Tsukuba, Tsukuba, Ibaraki, Japan

\section{Introduction}

Primary postpartum hemorrhage $(\mathrm{PPH})$ is the most common form of major obstetric hemorrhage and remains one of the major causes of maternal death in both developed and developing countries [1, 2]. In Japan, the maternal mortality rate was 3.1 per 100,000 births in 2008, and $\mathrm{PPH}$ is the second most common direct cause of death $[3,4]$. Among 151,866 women who delivered in 253 perinatal medical centers during 2012, 17.1\% experienced more than $1000 \mathrm{~mL}$ of blood loss, and $0.7 \%$ more than $3000 \mathrm{~mL}$ of blood loss [5]. The maternal mortality rate has been decreasing both in Japan and worldwide $[4,6]$, but an increasing trend in the rate of PPH was observed in developed countries including Australia, Canada, and the USA [7].

Once PPH has been identified, appropriate management is needed, which depends on the woman receiving care in a consultant-led maternity unit with access to laboratory and blood bank facilities and skilled obstetric and anesthetic staff readily available [2]. In Japan, approximately half of pregnant women give birth in hospitals, and other half give birth in clinics with one or two obstetricians [8]. These clinics do not have easy access to laboratory and blood bank facilities and skilled staff. In order to better manage PPH, it is important for clinicians to predict the occurrence and severity of PPH before delivery.

Some known risk factors for $\mathrm{PPH}$ are placenta previa, previous $\mathrm{PPH}$, Asian ethnicity, anemia, and preeclampsia. Recently, maternal coagulation and fibrinolysis profiles also have been investigated to predict severe postpartum hemorrhage. Although a correlation between the decrease of fibrinogen in the early phase of PPH and PPH severity has been reported $[9,10]$, there are few studies concerning the prediction of PPH occurrence before delivery. The aim of the present study was to determine effective predictive factors for PPH using a combination of antenatal coagulation and fibrinolysis profiles and maternal clinical demographics. 


\section{Materials and methods}

This study was conducted after approval of the institutional review board and Ethics Committee at the University of Tsukuba Hospital was obtained (number: H26-216).

\section{Participants}

We retrospectively reviewed the clinical records of all 1347 women who met the following three conditions: (1) provided written informed consent for participation in this study, (2) had antenatal care on and after gestational week (GW) 32 at our clinic, and (3) gave birth vaginally to singleton infants on and after GW 35 at our hospital during the 3-year study period from January 2011 to December 2013. During the study period, six blood parameters including hemoglobin concentration, platelet count, prothrombin time international normalized ratio (PT-INR), fibrinogen concentration, antithrombin (AT) activity, and D-dimer were determined twice at GW 29-32 (defined as mid-pregnancy in this study) and GW 35-37 (defined as pre-delivery). Finally, 1032 women with blood data available both at mid-pregnancy and pre-delivery were included in this study after excluding 315 women (Figure 1). All blood variables were determined at Tsukuba i-laboratory LLP immediately after sampling. Fibrinogen concentrations in plasma were determined with the Clauss method.

1347 women giving birth to singletons on and after gestational week (GW) 35 at our hospital during the study period.

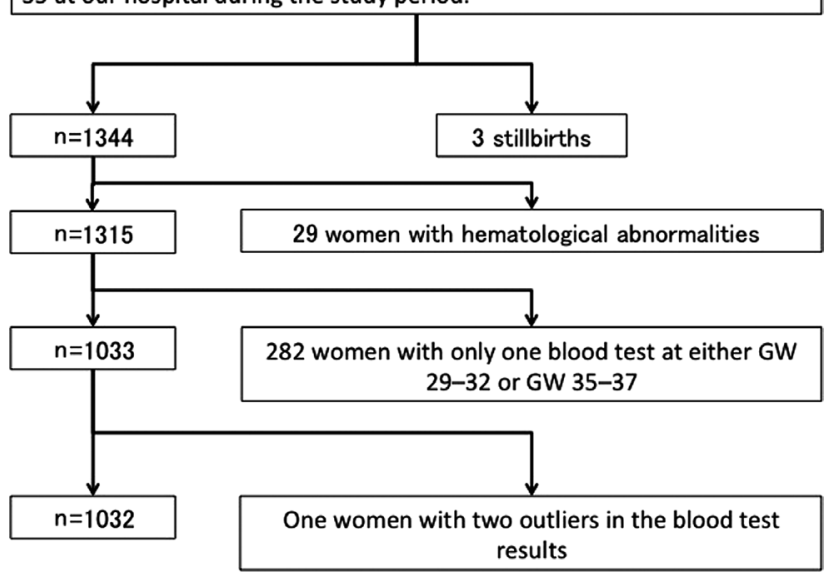

Figure 1: Flow diagram for participant selection.

Among 1347 candidates for this study, a total of 315 women were excluded from the present analysis: three with stillbirths; 29 with known hematologic abnormalities including 10 with idiopathic thrombocytopenic purpura, five with anti-phospholipid syndrome treated with low-dose aspirin, five with a history of thrombosis requiring anti-coagulation with heparin, two with myelodysplastic syndrome, two with protein S deficiency, and one each with familial antithrombin deficiency, aplastic anemia, thalassemia, recurrent miscarriage treated with aspirin, and transverse sinus thrombosis; 282 who underwent blood testing only once at either mid-pregnancy (gestational week 29-32) or pre-delivery (gestational week 35-37); and one with two outliers in the blood test results. The remaining 1032 women with available blood data from both the mid-pregnancy and pre-delivery periods were analyzed in this study.
Maternal clinical demographic data including body mass index (BMI, $\mathrm{kg} / \mathrm{m}^{2}$ ) on admission for childbirth, parity, complications of pregnancy, GW at delivery, and estimated blood loss (EBL) in the $2 \mathrm{~h}$ after delivery were collected from medical charts. PPH was defined as $\mathrm{EBL} \geq 700 \mathrm{~mL}$ in this study.

\section{Statistical analyses}

Data were analyzed using SPSS software version 22 (IBM). Student's $t$-test and the Mann-Whitney $U$-test were used for the comparison of means and median. The $\chi^{2}$ test and Fisher's exact test were used for the comparison of categorical data. Linear regression analysis was performed using Spearman's rank correlation coefficient. Categorized variables were converted to dummy variables for the univariate and multivariate logistic regression analyses. The cut-off levels for continuous variables included 35 years for maternal age, $28.2 \mathrm{~kg} / \mathrm{m}^{2}$ for BMI according to the definition of obese pregnant women proposed by the Japan Society for the Study of Obesity [11], 1.0 for PT-INR at both mid-pregnancy and pre-delivery (corresponding to approximately $75^{\text {th }}$ percentile values for both pregnancy stages in our population), $3.8 \mathrm{~g} / \mathrm{L}$ and $4.0 \mathrm{~g} / \mathrm{L}$ for fibrinogen at mid-pregnancy and pre-delivery, respectively (corresponding to approximately $15^{\text {th }}$ percentile values for each pregnancy stage in our population), $90 \%$ and $85 \%$ of normal activity level for AT activity at mid-pregnancy and pre-delivery, respectively (corresponding to approximately $15^{\text {th }}$ percentile values for each pregnancy stage in our population), and $1.9 \mu \mathrm{g} / \mathrm{mL}$ and $2.7 \mu \mathrm{g} / \mathrm{mL}$ for D-dimer at mid-pregnancy and pre-delivery, respectively (corresponding to approximately $75^{\text {th }}$ percentile values for each pregnancy stage in our population). Multivariate logistic regression analysis was used to determine independent risk factors for PPH and calculate adjusted odds ratio. In all analyses, P-values $<0.05$ were considered statistically significant.

\section{Results}

PPH occurred in 104 of 1032 women (10\%) (Table 1). The mean maternal age and antenatal BMI determined on admission for childbirth differed significantly between the two groups. A significantly larger number of women had a history of previous cesarean delivery in the PPH group than in the non-PPH group. Therefore, the three factors of maternal age, maternal BMI, and previous cesarean delivery were candidate independent risk factors for $\mathrm{PPH}$ among demographic characteristics.

Blood test results were compared between the two groups with and without PPH (Table 2). Among six blood variables, the mean AT activity level was significantly and consistently lower and the mean D-dimer level was significantly and consistently higher in the PPH group than in the non-PPH group at both mid-pregnancy and pre-delivery (Table 2). In addition, the number of women with lower fibrinogen levels, lower AT activity levels, and higher D-dimer levels was consistently and significantly 
Table 1: Clinical characteristics of the two groups.

\begin{tabular}{|c|c|c|c|c|c|}
\hline \multirow[b]{2}{*}{ Maternal age (years) } & \multicolumn{2}{|c|}{ PPH group $(n=104)$} & \multicolumn{2}{|c|}{ Non-PPH group $(n=928)$} & \multirow{2}{*}{$\frac{\text { P-value }}{0.036}$} \\
\hline & 34 & [19-43] & 32 & {$[14-47]$} & \\
\hline Antenatal BMI $\left(\mathrm{kg} / \mathrm{m}^{2}\right)$ & 26.1 & {$[20.1-51.2]$} & 25.2 & {$[18.2-52.5]$} & 0.001 \\
\hline Nulliparous & 62 & $(59.6 \%)$ & 503 & $(54.2 \%)$ & 0.293 \\
\hline Previous cesarean delivery & 12 & $(11.5 \%)$ & 59 & $(6.4 \%)$ & 0.048 \\
\hline \multicolumn{6}{|l|}{ Complications } \\
\hline $\mathrm{PIH}$ & 5 & $(4.8 \%)$ & 17 & $(1.8 \%)$ & 0.062 \\
\hline $\mathrm{DM} / \mathrm{GDM}$ & 21 & $(20.2 \%)$ & 186 & $(20.0 \%)$ & 0.971 \\
\hline Uterine fibroma & 4 & (3.8\%) & 64 & $(6.9 \%)$ & 0.234 \\
\hline
\end{tabular}

Data are presented as median [range], or number (\%).

$\mathrm{BMI}=$ Body mass index, $\mathrm{PIH}=$ pregnancy-induced hypertension including preeclampsia and gestational hypertension, $\mathrm{DM} / \mathrm{GDM}=$ diabetes mellitus/gestational diabetes mellitus.

Table 2: Comparison of blood test results between the two groups.

\begin{tabular}{|c|c|c|c|c|c|}
\hline & \multicolumn{2}{|c|}{ PPH group $(n=104)$} & \multicolumn{2}{|c|}{ Non-PPH group $(n=928)$} & \multirow[t]{2}{*}{ P-value } \\
\hline & Mean \pm SD or median & range & Mean \pm SD or median & range & \\
\hline \multicolumn{6}{|c|}{ Mid-pregnancy (GW 29-32) } \\
\hline Hemoglobin (g/dL) & $11.0 \pm 0.96$ & $9.1-13.3$ & $11.2 \pm 0.85$ & $8.1-14.4$ & 0.596 \\
\hline Platelets $\left(10^{9} / \mathrm{L}\right)$ & $228.7 \pm 63.4$ & $96-390$ & $225.7 \pm 49.8$ & $88-439$ & 0.647 \\
\hline PT-INR & $0.98 \pm 0.06$ & $0.84-1.21$ & $0.98 \pm 0.05$ & $0.84-1.28$ & 0.160 \\
\hline Fibrinogen $(\mathrm{g} / \mathrm{L})$ & $4.55 \pm 0.89$ & $2.76-7.17$ & $4.54 \pm 0.75$ & $2.47-7.93$ & 0.945 \\
\hline AT activity (\%) & $99.1 \pm 11.0$ & $72.0-132.2$ & $101.3 \pm 10.1$ & $72.5-142.1$ & 0.040 \\
\hline D-dimer $(\mu \mathrm{g} / \mathrm{mL})$ & 1.7 & $0.7-9.8$ & 1.4 & $0.4-9.2$ & $<0.001^{\mathrm{a}}$ \\
\hline \multicolumn{6}{|c|}{ Pre-delivery (GW 35-37) } \\
\hline Hemoglobin (g/dL) & $11.1 \pm 0.89$ & $9.1-14.2$ & $11.1 \pm 0.86$ & $8.3-15.1$ & 0.778 \\
\hline Platelets $\left(10^{9} / \mathrm{L}\right)$ & $220.8 \pm 60.6$ & $35-371$ & $221.5 \pm 52.3$ & $73-443$ & 0.918 \\
\hline PT-INR & $0.97 \pm 0.05$ & $0.87-1.14$ & $0.96 \pm 0.05$ & $0.79-1.26$ & 0.120 \\
\hline Fibrinogen $(\mathrm{g} / \mathrm{L})$ & $4.64 \pm 0.82$ & $3.10-6.63$ & $4.73 \pm 0.74$ & $2.70-7.55$ & 0.258 \\
\hline AT activity (\%) & $96.2 \pm 11.5$ & $59.4-126.6$ & $98.3 \pm 10.8$ & $66.7-141.4$ & 0.060 \\
\hline D-dimer $(\mu \mathrm{g} / \mathrm{mL})$ & 2.2 & $0.8-11.6$ & 1.9 & $0.6-12.8$ & $0.001^{\mathrm{a}}$ \\
\hline
\end{tabular}

Mean and median values were compared between the two groups using Student's $t$-test and amn-Whitney $U$-test, respectively. $\mathrm{GW}=\mathrm{Gestational}$ week, $\mathrm{PT}-\mathrm{INR}=$ prothrombin time international normalized ratio, $\mathrm{AT}=$ antithrombin.

greater in the PPH group than in the non-PPH group at both mid-pregnancy and pre-delivery (Table 3 ). The number of women $\geq 35$ years of age, with BMI $>28.2 \mathrm{~kg} / \mathrm{m}^{2}$, and with previous cesarean delivery was significantly greater in the PPH group than in the non-PPH group (Table 3). Thus, nine factors including maternal age $\geq 35$ years, maternal BMI $>28.2 \mathrm{~kg} / \mathrm{m}^{2}$, previous cesarean delivery, fibrinogen $<3.8 \mathrm{~g} / \mathrm{L}$ at mid-pregnancy and $<4.0 \mathrm{~g} / \mathrm{L}$ at predelivery, AT activity $<90 \%$ at mid-pregnancy and $<85 \%$ at pre-delivery, and D-dimer $\geq 1.9 \mu \mathrm{g} / \mathrm{mL}$ at mid-pregnancy and $\geq 2.7 \mu \mathrm{g} / \mathrm{mL}$ at pre-pregnancy were candidate independent risk factors for PPH.

As expected, a strong correlation was confirmed between the two data sets concerning fibrinogen levels, AT activity levels, and D-dimer levels determined at mid-pregnancy and pre-delivery; correlation coefficients $(r)$ were
0.714 for fibrinogen levels, 0.76 for AT activity levels, and 0.873 for D-dimer levels determined at the two pregnancy stages. However, no significant correlations were observed among these three blood variables at mid-pregnancy as well as pre-delivery period. Fibrinogen and D-dimer increased significantly, and AT activity decreased significantly with advancing gestation (all P-values $<0.001$ ). Therefore, multivariate logistic regression analysis was performed separately for the mid-pregnancy and pre-delivery data. In both analyses, three factors including maternal age $\geq 35$ years (Yes or No), BMI $>28.2 \mathrm{~kg} / \mathrm{m}^{2}$ (Yes or No), and previous cesarean delivery (Yes or No) were entered. In addition, fibrinogen $<3.8 \mathrm{~g} / \mathrm{L}$ at mid-pregnancy (Yes or No), AT activity $<90 \%$ at mid-pregnancy (Yes or No), and D-dimer $\geq 1.9 \mu \mathrm{g} / \mathrm{mL}$ at mid-pregnancy (Yes or No) were entered in the first analysis, and fibrinogen $<4.0 \mathrm{~g} / \mathrm{L}$ at 
Table 3: Categorized characteristics in the two groups.

\begin{tabular}{|c|c|c|c|c|c|}
\hline \multirow[b]{2}{*}{ Maternal age $\geq 35$} & \multicolumn{2}{|c|}{ PPH group $(n=104)$} & \multicolumn{2}{|c|}{ Non-PPH group $(n=928)$} & \multirow{2}{*}{$\frac{\text { P-value }}{0.033}$} \\
\hline & 48 & $(46)$ & 330 & $(36)$ & \\
\hline $\mathrm{BMI}>28.2$ & 29 & $(28)$ & 185 & $(20)$ & 0.058 \\
\hline Nulliparous & 62 & $(60)$ & 505 & $(54)$ & 0.293 \\
\hline Previous cesarean delivery & 12 & (12) & 59 & (6) & 0.048 \\
\hline \multicolumn{6}{|l|}{ Mid-pregnancy (GW 29-32) } \\
\hline PT-INR $\geq 1.0$ & 39 & (38) & 306 & (33) & 0.354 \\
\hline Fibrinogen $<3.8 \mathrm{~g} / \mathrm{L}$ & 26 & $(25)$ & 139 & (15) & 0.008 \\
\hline AT activity $<90 \%$ & 22 & $(21)$ & 120 & (13) & 0.021 \\
\hline D-dimer $\geq 1.9 \mu \mathrm{g} / \mathrm{mL}$ & 46 & (44) & 234 & $(25)$ & $<0.001$ \\
\hline \multicolumn{6}{|l|}{ Pre-delivery (GW 35-37) } \\
\hline PT-INR $\geq 1.0$ & 30 & $(29)$ & 207 & $(22)$ & 0.133 \\
\hline Fibrinogen $<4.0 \mathrm{~g} / \mathrm{L}$ & 26 & $(25)$ & 139 & (15) & 0.008 \\
\hline AT activity $<85 \%$ & 20 & (19) & 94 & $(10)$ & 0.005 \\
\hline D-dimer $\geq 2.7 \mu \mathrm{g} / \mathrm{mL}$ & 36 & (35) & 198 & $(21)$ & 0.002 \\
\hline
\end{tabular}

Number of women (percentage) is indicated.

$\mathrm{BMI}=$ Body mass index, GW=gestational week, $\mathrm{PT}-\mathrm{INR}=$ prothrombin time international normalized ratio, $\mathrm{AT}=$ antithrombin .

pre-delivery (Yes or No), AT activity $<85 \%$ at pre-delivery (Yes or No), and D-dimer $\geq 2.7 \mu \mathrm{g} / \mathrm{mL}$ at pre-delivery (Yes or No) were entered in the second analysis. In both analyses, maternal age $\geq 35$ years, BMI $>28.2 \mathrm{~kg} / \mathrm{m}^{2}$, and previous cesarean delivery were identified as independent risk factors for $\mathrm{PPH}$, increasing the risk of $\mathrm{PPH}$ by approximately $70 \%, 100 \%$, and $180 \%$, respectively (Table 4). Fibrinogen $<3.8 \mathrm{~g} / \mathrm{L}$ and D-dimer $\geq 1.9 \mu \mathrm{g} / \mathrm{mL}$ at mid-pregnancy were independent risk factors for PPH, and all three blood variables, fibrinogen $<4.0 \mathrm{~g} / \mathrm{L}$, AT activity $<85 \%$, and D-dimer $\geq 2.7 \mu \mathrm{g} / \mathrm{mL}$ at pre-delivery, were significant independent risk factors for $\mathrm{PPH}$, increasing the risk of PPH by approximately 100\%, 80\%, and $100 \%$, respectively (Table 4).

Participants were divided into quintile groups according to fibrinogen, AT activity, and D-dimer levels at GW 35-37 (Figure 2). As expected, the PPH prevalence rate was highest in the $1^{\text {st }}$ quintile groups of fibrinogen and AT activity levels and the $5^{\text {th }}$ quintile group of D-dimer level.

\section{Discussion}

To our knowledge, this study demonstrated for the first time that three blood variables, fibrinogen, AT activity, and D-dimer, in late gestation were independent risk factors for PPH. D-dimer level as well as fibrinogen level in mid-pregnancy were also independent risk factors for $\mathrm{PPH}$ in this study. Maternal age $\geq 35$, BMI $>28.2 \mathrm{~kg} / \mathrm{m}^{2}$, and history of cesarean delivery were confirmed as risk factors, as reported previously by several studies [12-17].
Table 4: Results of multivariate logistic regression analyses.

\begin{tabular}{lrr}
\hline & aOR $(95 \% \mathrm{Cl})$ & P-value \\
\hline Mid-pregnancy (GW 29-32) & & \\
Age $\geq 35$ years & $1.77(1.16-2.71)$ & 0.009 \\
BMI $>28.2 \mathrm{~kg} / \mathrm{m}^{2}$ & $2.02(1.24-3.28)$ & 0.004 \\
Previous cesarean & $2.91(1.37-6.18)$ & 0.005 \\
Fibrinogen $<3.8 \mathrm{~g} / \mathrm{L}$ & $1.83(1.09-3.07)$ & 0.022 \\
AT activity $<90 \%$ & $1.62(0.94-2.79)$ & 0.081 \\
D-dimer $\geq 1.9 \mu \mathrm{g} / \mathrm{mL}$ & $2.59(1.68-3.99)$ & $<0.001$ \\
Pre-delivery $(\mathrm{GW} 35-37)$ & & \\
Age $\geq 35$ years & $1.75(1.15-2.68)$ & 0.009 \\
BMI $>28.2 \mathrm{~kg} / \mathrm{m}^{2}$ & $1.95(1.20-3.16)$ & 0.007 \\
Previous cesarean & $2.77(1.31-5.83)$ & 0.007 \\
Fibrinogen $<4.0 \mathrm{~g} / \mathrm{L}$ & $1.96(1.18-3.27)$ & 0.010 \\
AT activity $<85 \%$ & $1.84(1.05-3.21)$ & 0.032 \\
D-dimer $\geq 2.7 \mu \mathrm{g} / \mathrm{mL}$ & $2.03(1.29-3.19)$ & 0.002 \\
\hline
\end{tabular}

aOR $(95 \% \mathrm{Cl})=$ Adjusted odds ratio ( $95 \%$ confidence interval), $\mathrm{BMI}=$ body mass index, $\mathrm{GW}=$ gestational week, $\mathrm{AT}=$ antithrombin.

The D-dimer level increased significantly with advancing gestation in this study, consistent with results of previous studies [18]. Therefore, in the present study, different cut-off levels for D-dimer were set in the two periods, i.e. $\geq 1.9 \mu \mathrm{g} / \mathrm{mL}$ at mid-pregnancy and $\geq 2.7 \mu \mathrm{g} / \mathrm{mL}$ at predelivery, corresponding to the $73^{\text {rd }}(752 / 1032$, Table 3$)$ and $77^{\text {th }}$ (798/1032, Table 3) percentile values, respectively, in our population. These cut-off levels predicted the risk of PPH independently of other risk factors. Higher D-dimer level is seen at the early stage of PPH [9]. However, to our knowledge, no previous studies have focused on the association between antenatal D-dimer levels and PPH occurrence. 

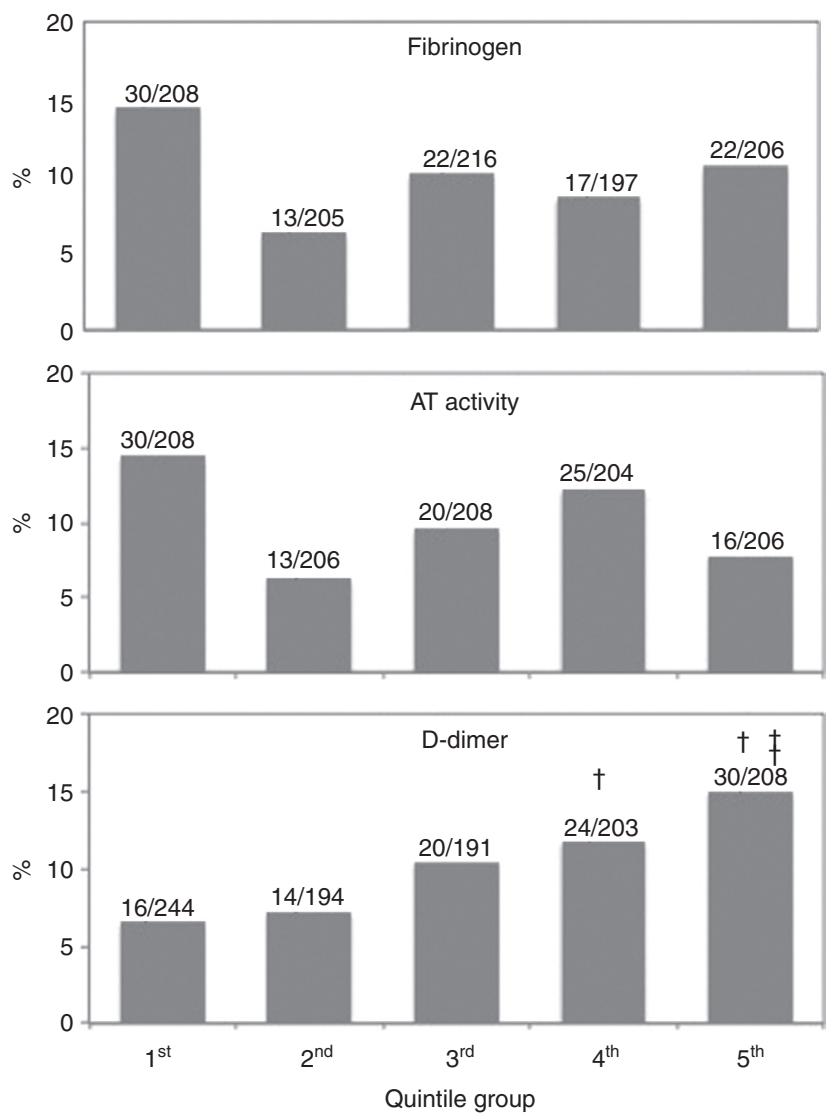

Figure 2: Prevalence rate of PPH in the quintile groups divided by fibrinogen, AT activity, and D-dimer levels at gestational week 35-37.

One thousand and thirty-two women were divided into quintile groups by fibrinogen level at GW 35-37 (upper panel), AT activity level at gestational week 35-37 (middle panel), and D-dimer level at gestational week 35-37 (lower panel). The cut-off values for quintiles were 4.12, 4.50, 4.88, and $5.36 \mathrm{~g} / \mathrm{L}$ for fibrinogen; 89 , 95,100 , and $107 \%$ for AT activity, and 1.5, 1.8, 2.2, and $2.9 \mu \mathrm{g} / \mathrm{mL}$ for D-dimer. The actual number of women in each quintile is indicated above each bar. The Jonckheere- Terpstra trend test indicated that quintile groups included different groups with respect to both the distribution of EBL and the prevalence rate of $P P H(P<0.001)$; $\dagger, P<0.05$ vs. the $1^{\text {st }}$ quintile group with respect to $E B L . \neq, P<0.05$ vs. the $1^{\text {st }}$ quintile group with respect to the prevalence rate of $\mathrm{PPH}$.

It was expected that higher D-dimer was a risk factor of PPH. A significant negative correlation is seen between fibrinogen and D-dimer levels in late twin gestation, in which an exaggerated coagulation response has been suggested [19] based on the finding that D-dimer level is significantly higher and fibrinogen is significantly lower in late twin gestation than in late singleton gestation $[18,19]$. In addition, a low fibrinogen level at the initial phase of PPH increases the risk of severe PPH $[9,10]$, and low antenatal fibrinogen level increases the risk of PPH [20, 21], as was confirmed in this study. Thus, as the increase in
D-dimer may have reflected enhanced fibrinolysis, i.e. consumption of fibrinogen leading to lower fibrinogen level, higher D-dimer level was expected to be a risk factor for PPH. An increase of fibrinogen up to two-fold of the non-pregnant level [19] may be a physiologic adaption to hyperconsumption of fibrinogen occurring in pregnancy, as evidenced by higher D-dimer levels in pregnant women than in non-pregnant women [18]. However, no significant correlation was seen between fibrinogen and D-dimer levels in this study, consistent with results of the previous study in singleton pregnancies by Yamada et al. [19]. Even in the presence of fibrinogen hyperconsumption, a low fibrinogen level theoretically does not occur when the production of fibrinogen compensates its consumption. The absence of a significant correlation between fibrinogen and D-dimer levels in late singleton gestation may be explained by milder hyperconsumption of fibrinogen in singleton pregnancies compared to that in twin pregnancies.

AT activity decreased significantly with advancing gestation in this study, consistent with the results of a previous study [22], and was an independent risk factor for $\mathrm{PPH}$ in this study. As AT is the most important molecule for anti-coagulation in the circulating blood, combining with thrombin at a 1:1 ratio, the decreased AT activity may suggest exaggerated thrombin generation. Blood coagulation occurs in the presence of thrombin. Thus, both the lower AT activity and higher D-dimer level occur as a result of enhanced coagulation. Our findings that higher D-dimer level, lower fibrinogen level, and lower AT activity level were risk factors for PPH imply that enhanced coagulation leading to lower levels of fibrinogen, AT activity, and/or D-dimer increased the risk of PPH. We speculate that some women with such characteristics appearing in blood test results may have experienced additional blood loss to the usual blood loss, thus reaching EBL meeting the criteria for PPH due to hematologic problems associated with the hypercoagulable state in pregnancy.

In this study, advanced maternal age ( $\geq 35$ years) and meeting the Japanese obesity threshold (BMI $>28.2 \mathrm{~kg} / \mathrm{m}^{2}$ ) immediately before childbirth were independent risk factors of PPH, consistent with results of previous studies [12-15]. Previous cesarean delivery was also an independent risk factor of $\mathrm{PPH}$, perhaps due to mechanical problems with respect to uterine contraction after vaginal delivery.

This study has some limitations. First, University of Tsukuba Hospital is one of the largest perinatal medical centers in Ibaraki prefecture, so cases largely involved complicated pregnancies and primiparous women of advanced maternal age. Second, the ethnic distribution of patients was biased toward Asian and Hispanic, and these 
ethnicities are risk factors of uterine atony $[2,15]$. Third, women who did not have regular antenatal checkups were excluded, and this group is considered to be at high risk for PPH. Finally, the cost effectiveness of prenatal screening of D-dimer or fibrinogen solely for the prediction of PPH might be controversial.

\section{Conclusion}

This study demonstrated higher D-dimer level as well as lower fibrinogen and AT activity levels in late gestation were independent risk factors for PPH. Although the precise mechanisms leading to PPH remain unclear at present, our results suggested that a state of enhanced coagulation leading to higher D-dimer level, lower AT activity level, and/or lower fibrinogen level contributed to increased risk of PPH in some pregnant women with such characteristics on blood test results. Term pregnant women found incidentally to have such a status with enhanced coagulation should be cared with extra caution against PPH.

Disclosure: We have no circumstances of conflict of interest that should be disclosed. We have not received any financial support regarding this study.

\section{References}

[1] Khan KS, Wojdyla D, Say L, Gülmezoglu AM, Van Look PFA. WHO analysis of causes of maternal death: a systematic review. Lancet. 2006;367:1066-74.

[2] Royal College of Obstetricians and Gynaecologists. Postpartum haemorrhage, prevention and management (Green-top Guideline No. 52) [Internet] 2009.

[3] Japan Society of Obstetrics and Gynecology, The report of 83 cases of maternal mortality in 2010-2012 [Internet] 2013. Available from: http://www.jaog.or.jp/all/document/67_130710.pdf (Japanese).

[4] Japanese Ministry of Health, Labour and Welfare. Change in maternal mortality rate in Japan [Internet]. Available from: http://www.mhlw.go.jp/english/database/db-hh/xls/2-18.xls.

[5] Japan Society of Obstetrics and Gynecology, The report of the prenatal committee in 2013 [Internet] 2014. Available from: http://fa.kyorin.co.jp/jsog/readPDF.php?file=66/6/066061450. pdf (Japanese).

[6] United Nations Population Fund. By choice, not by chance: family planning, human rights and development. New York: UNFPA; 2012.

[7] Knight M, Callaghan WM, Berg C, Alexander S, Bouvier-Colle MH, Ford JB, et al. Trends in postpartum hemorrhage in high resource countries: a review and recommendations from the International Postpartum Hemorrhage Collaborative Group. BMC Pregnancy Childbirth. 2009;9:55.
[8] Japanese Ministry of Health, Labour and Welfare. Vital statistics of population. [Internet] 2013. Available from: http://www.mhlw.go.jp/toukei/saikin/hw/hoken/kiso/21.html (Japanese).

[9] Charbit B, Mandelbrot L, Samain E, Baron G, Haddaoui B, Keita $\mathrm{H}$, et al. The decrease of fibrinogen is an early predictor of the severity of postpartum hemorrhage. J Thromb Haemost. 2007;5:266-73.

[10] Cortet M, Deneux-Tharaux C, Dupont C, Colin C, Rudigoz RC, Bouvier-Colle $\mathrm{MH}$, et al. Association between fibrinogen level and severity of postpartum haemorrhage: secondary analysis of a prospective trial. Br J Anaesth. 2012;108:984-9.

[11] Japan Society for the Study of Obesity. Guidelines for the management of obesity disease 2006. Himan Kenkyu. 2006;12(suppl):1-91. [Japanese].

[12] Matsuda Y, Kawamichi Y, Hayashi K, Shiozaki A, Satoh S, Saito S. Impact of maternal age on the incidence of obstetrical complications in Japan. J Obstet Gynaecol Res. 2011;37: 1409-14.

[13] Jolly M, Sebire N, Harris J, Robinson S, Regan L. The risks associated with pregnancy in women aged 35 years or older. Hum Reprod. 2000;15:2433-7.

[14] Magann EF, Doherty DA, Sandlin AT, Chauhan SP, Morrison JC. The effects of an increasing gradient of maternal obesity on pregnancy outcomes. Aust N Z J Obstet Gynaecol. 2013;53:250-7.

[15] Wetta LA, Szychowski JM, Seals S, Mancuso MS, Biggio JR, Tita AT. Risk factors for uterine atony/postpartum hemorrhage requiring treatment after vaginal delivery. Am J Obstet Gynecol. 2013;209:51.e1-6.

[16] Ford JB, Roberts CL, Simpson JM, Vaughan J, Cameron CA. Increased postpartum hemorrhage rates in Australia. Int J Gynaecol Obstet. 2007;98:237-43.

[17] Joseph KS, Rouleau J, Kramer MS, Young DC, Liston RM, Baskett TF, et al. Investigation of an increase in postpartum haemorrhage in Canada. Br J Obstet Gynaecol. 2007;114: 751-9.

[18] Yamada T, Kawaguchi S, Araki N, Takeda M, Nishida R, Yamada $\mathrm{T}$, et al. Difference in the D-dimer rise between women with singleton and multifetal pregnancies. Thromb Res. 2013;131: 493-6.

[19] Yamada T, Morikawa M, Yamada T, Akaishi R, Kojima T, Minakami H. Fibrinogen levels in the late stage of twin pregnancy. Thromb Res. 2015;135:318-21.

[20] Simon L, Santi TM, Sacquin P, Hamza J. Pre-anaesthetic assessment of coagulation abnormalities in obstetric patients; usefulness, timing and clinical implications. $\mathrm{Br} J$ Anaesth. 1997;78:678-83.

[21] Yamada T, Akaishi R, Oda Y, Nishida R, Yamada T, Ishikawa S, et al. Antenatal fibrinogen concentrations and postpartum haemorrhage. Int J Obstet Anesth. 2014;23:365-70.

[22] Tsunoda T, Ohkuchi A, Izumi A, Watanabe T, Matsubara S, Sato I, et al. Antithrombin III activity and platelet count are more likely to decrease in twin pregnancies than in singleton pregnancies. Acta Obstet Gynecol Scand. 2002;81:840-5.

The authors stated that there are no conflicts of interest regarding the publication of this article. 